\title{
X-ray phase-contrast imaging with an Inverse Compton Scattering source
}

Cite as: AIP Conference Proceedings 1266, 39 (2010); https://doi.org/10.1063/1.3478194

Published Online: 22 July 2010

M. Endrizzi, M. Carpinelli, P. Delogu, P. Oliva, B. Golosio, T. E. Gureyev, U. Bottigli, and A. Stefanini

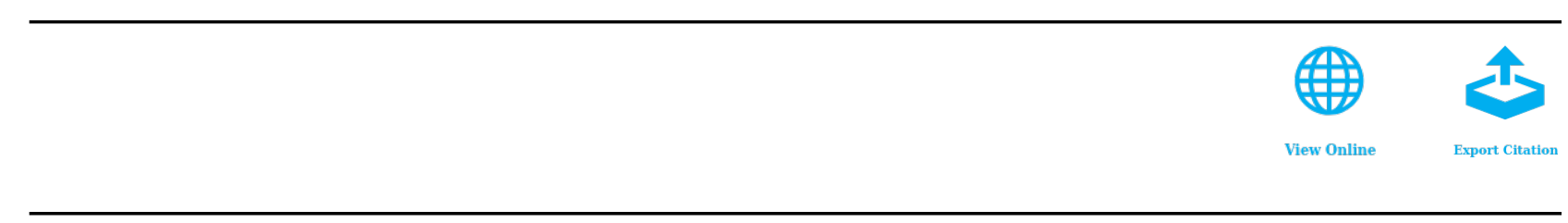

\section{ARTICLES YOU MAY BE INTERESTED IN}

High duty cycle inverse Compton scattering X-ray source

Applied Physics Letters 109, 253504 (2016); https://doi.org/10.1063/1.4972344

Quantitative evaluation of single-shot inline phase contrast imaging using an inverse compton x-ray source

Applied Physics Letters 97, 134104 (2010); https://doi.org/10.1063/1.3491430

Phase-contrast imaging with a novel $\mathrm{X}$-ray source

AIP Conference Proceedings 1221, 119 (2010); https://doi.org/10.1063/1.3399237

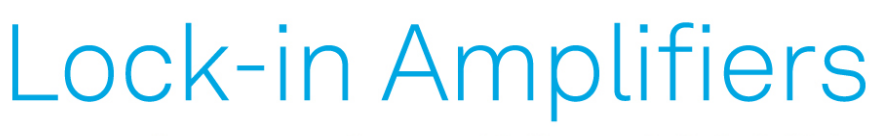

... and more, from DC to $600 \mathrm{MHz}$

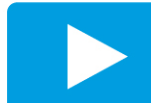

Watch 


\title{
X-ray phase-contrast imaging with an Inverse Compton Scattering source
}

\author{
M.Endrizzi ${ }^{* \dagger}$, M. Carpinelli**, P. Delogu ${ }^{\ddagger}$, P. Oliva**, B. Golosio**, T. E. Gureyev ${ }^{\dagger}$, \\ U. Bottigli* and A. Stefanini ${ }^{*}$ \\ ${ }^{*}$ Dip. di Fisica, Università di Siena, Italy and Istituto Nazionale di Fisica Nucleare INFN, Sezione di Pisa, Italy \\ ${ }^{\dagger}$ CSIRO, Materials Science and Engineering, Clayton South, 3169, VIC, Australia \\ ${ }^{*}$ Strutt. Dip. di Matematica e Fisica, Università degli Studi di Sassari, Italy and Istituto Nazionale di Fisica \\ Nucleare INFN, Sezione di Cagliari, Italy \\ "Dip. di Fisica "E. Fermi", Università di Pisa, Italy and Istituto Nazionale di Fisica Nucleare INFN, Sezione di
}

Pisa, Italy

\begin{abstract}
Single-shot in-line phase-contrast imaging with the Inverse Compton Scattering X-ray source available at ATF (Accelerator Test Facility) at Brookhaven National Laboratory is experimentally demonstrated. Phase-contrast images of polymer wires are obtained with a single X-ray pulse whose time length is about 1 picosecond. The edge-enhancement effect is clearly visible in the images and simulations show a quantitative agreement with experimental data. A phase-retrieval step in the image processing leads to a accurate estimation of the projected thickness of our samples. Finally, a single-shot image of a wasp is presented as an example of a biological sample.
\end{abstract}

Keywords: Inverse Compton Scattering, X-ray imaging, phase-contrast imaging

PACS: 87.59 .-e

\section{INTRODUCTION}

Inverse Compton Scattering (ICS) sources are capable of producing very short X-ray pulses characterized by high flux and brightness, tunable energy, quasimonochromatic spectrum and small spot size. These properties can be exploited to realize high-quality Xray imaging of particular interest for the biomedical field $[1,2,3]$. An application to in-line phase contrast imaging is here presented.

\section{EXPERIMENTAL SET-UP}

Figure 1 depicts our experiment layout: the X-ray are generated inside the Compton chamber by a head-on collision of a bunch of relativistic electrons with an intense laser pulse. After the interaction the electron beam is deflected by a dipole magnet into the beam stopper while the X-ray beam exits through the Be window and reaches the sample. A helium-filled pipe minimizes the intensity losses due to absorption while the beam propagates towards the X-ray detector. For a more accurate description of the beamline we refer the reader to a previous work [5].

The X-ray detector is a CMOS sensor coated with $165 \mu \mathrm{m}$ of directly deposited microcolumnar CsI (Hamamatsu $C 9728 D K-10$ ). The pixel size is $50 \times 50 \mu \mathrm{m}$ for a total active area of about $5 \times 5 \mathrm{~cm}$. The detector has

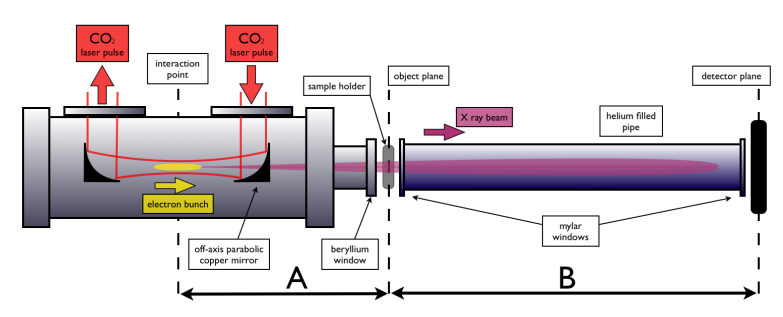

FIGURE 1. Sketch of the experiment (not to scale), $A=105$ $\mathrm{cm}$ and $B=223.5 \mathrm{~cm}$.

been characterized with respect to its Line Spread Function and energy response in order to perform quantitative measurements.

TABLE 1. X-ray beam parameters.

\begin{tabular}{rl}
\hline \multicolumn{2}{c}{ X-ray beam $[4,5,6]$} \\
\hline photons/pulse & $2 \cdot 10^{8}$ \\
Energymax & $10.4 \mathrm{keV}$ \\
spread $_{\min }$ & $0.6 \%$ \\
divergence $^{2}$ & $5 \mathrm{mrad}$ \\
pulse length & $\sim 1 \mathrm{ps}$ \\
\hline
\end{tabular}

Table 1 summarizes the parameters of the X-ray beam. The estimated number of photons scattered into $1 \mathrm{mrad}^{2}$ to $\approx 2 \cdot 10^{6}$ per pulse. Keeping in mind that this is a single-shot experiment, the flux density is approximately $7 \cdot 10^{16} \mathrm{ph} . \mathrm{s}^{-1} \mathrm{mrad}^{-2}(0.1 \% \mathrm{bw})^{-1}$. 


\section{RESULTS}

A single-shot exposure of a PET (polyethylene terephthalate) wire $520 \mu \mathrm{m}$ thick is shown in figure 2 ; the image is only corrected for the dark current. The intensity distribution without the object is estimated with a twodimensional Gaussian fit using all the pixels that do not contain any sample detail. With this approximation to the flat-field the raw image can be equalized. In figure 3 the PET sample and a PMMA (polymethyl methacrylate) wire with diameter $1124 \mu \mathrm{m}$ are shown. The fitting procedure for the flat-field is required due the shot-to-shot fluctuations of the beam (intensity, position and shape).

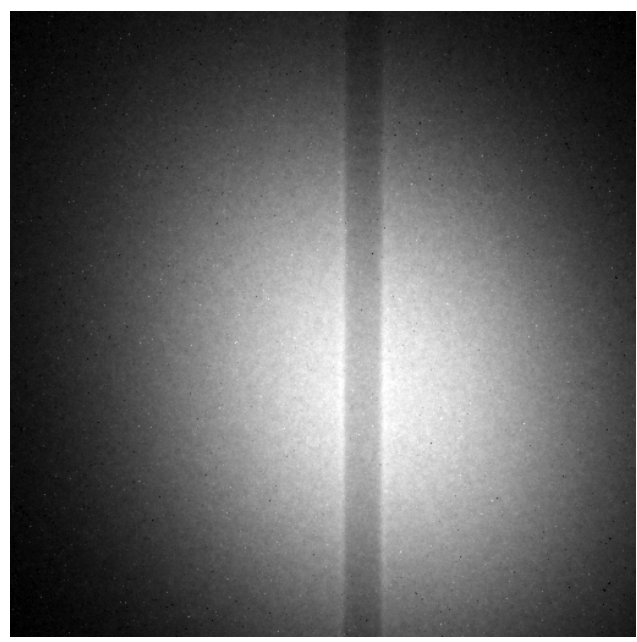

FIGURE 2. Raw single-shot image

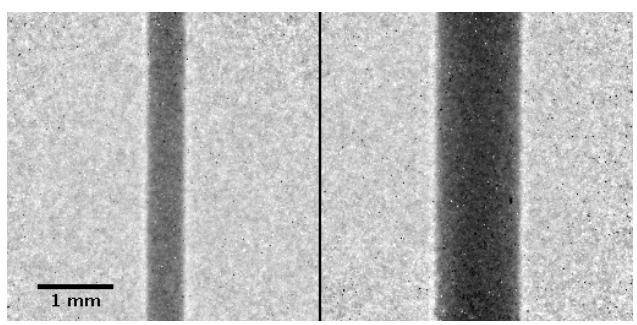

FIGURE 3. Ffit-based image equalization.
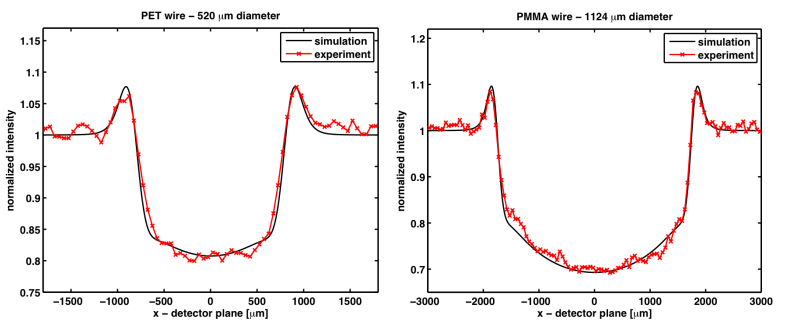

FIGURE 4. Comparison with simulation for the PET $520 \mu \mathrm{m}$ diameter sample (left) and for the PMMA $1124 \mu \mathrm{m}$ (right).
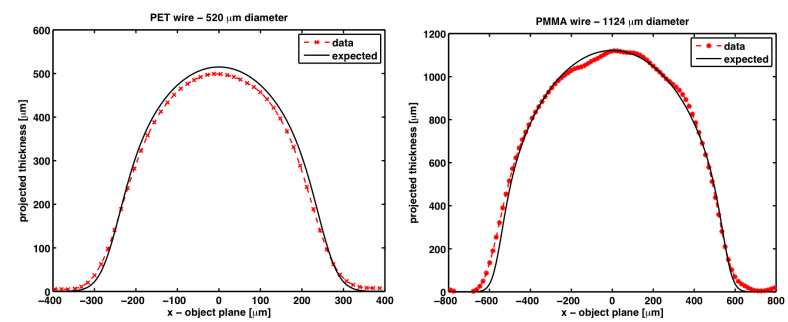

FIGURE 5. Projected thickness at the object plane, PET (left) and PMMA (right) samples.

A simulation [7] is compared to the normalized intensity profile (fig. 4). The source is modeled as a superposition of independent point sources, Gaussian distributed with a Full Width Half Maximum of $80 \mu \mathrm{m}$. The spectrum is chosen as quasi-monochromatic with mean energy $10.4 \mathrm{keV}$ and standard deviation $0.04 \mathrm{keV}$. The detector Line Spread Function is modeled with two Gaussian functions $(\sigma 93$ and $238 \mu \mathrm{m}$ relative amplitude 0.4 ). The normalized intensity profile is then processed [9] with a phase-retrieval algorithm [8] based on the monochromatic TIE for a homogeneous object. From the phase-retrieved image the projected thickness of the wires can be accurately estimated as shown in figure 5 .

A single-shot image of a wasp is shown in figure 6. It contains both absorption and phase contrast, absorption dominates in the central part of the sample while phase effects are visible at the edges of the legs, mesosoma and wing. The noise pattern present at the corners is a consequence of the intensity fall-out.

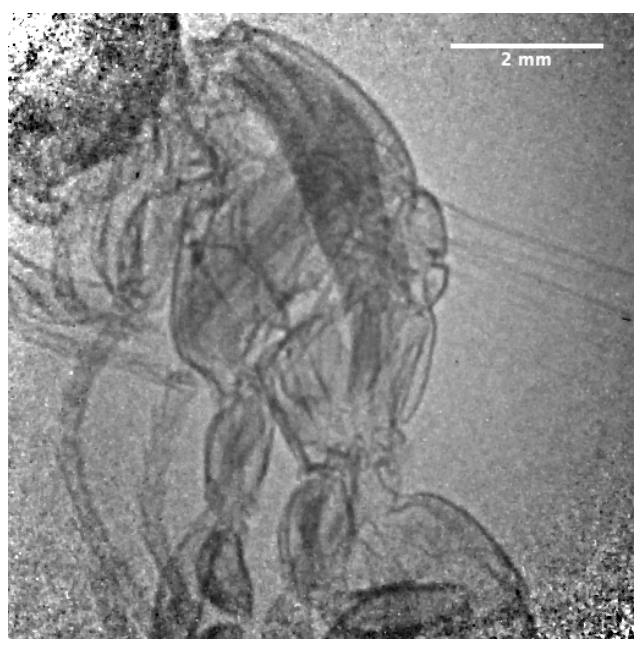

FIGURE 6. Single-shot image of a wasp. 


\section{CONCLUSION}

Single-shot phase-constrast imaging has been experimentally demonstrated with an Inverse Compton Scattering X-ray source using in-line geometry on a very short time scale. The edge-enhancement effects are clearly visible in the images and a preliminary analysis of homogeneous samples shows quantitative agreement with the simulations. These results are encouraging for future application of this kind of sources to X-ray biomedical imaging.

\section{ACKNOWLEDGMENTS}

We would like to acknowledge the staff at ATF, in particular V. Yakimenko, J. Park and I. Pogorelsky.

\section{REFERENCES}

1. Ikeura-Sekiguchi et al. Appl. Phys. Lett. 92, 131107 (2008).

2. P. Oliva et al. Med. Phys. 36,(11) 5149-5161 (2009).

3. P. Oliva et al. NIM A 615 (2010) 93Đ99

4. E. Esarey et al. Phys. Rev. E 48, 3003 (1993).

5. I. V. Pogorelsky et al. Phys. Rev. STAB 3, 090702 (2000).

6. W. J. Brown, F. Hartemann. Phys. Rev. ST AB, 7, 060703, (2004).

7. B. Golosio et al. J. Appl. Phys. 104, 093102 (2008).

8. D. Paganin et al.J. Microsc. 206, 33-40 (2002).

9. www.ts-imaging.net/Services/AppInfo/X-TRACT.aspx. 